\title{
Cometary ion dynamics observed in the close vicinity of comet 67P/Churyumov-Gerasimenko during the intermediate activity period
}

\author{
L. Berčič ${ }^{1,2}$, E. Behar ${ }^{2,1}$, H. Nilsson ${ }^{2,1}$, G. Nicolaou ${ }^{2,4}$, G. Stenberg Wieser ${ }^{2}$, M. Wieser ${ }^{2}$, and C. Goetz ${ }^{3}$ \\ ${ }^{1}$ Luleå University of Technology, Kiruna, Rymdcampus 1, 98192 Kiruna, Sweden \\ ${ }^{2}$ Swedish Institute of Space Physics, Rymdcampus 1, 98192 Kiruna, Sweden \\ e-mail: laura.bercic@irf.se \\ 3 Technische Universität Braunschweig, Institute for Geophysics and Extraterrestrial Physics, Mendelssohnstraße 3, \\ 38106 Braunschweig, Germany \\ ${ }^{4}$ Mullard Space Science Laboratory, University College London, Surrey, UK
}

Received 11 October 2017 / Accepted 21 January 2018

\begin{abstract}
Aims. Cometary ions are constantly produced in the coma, and once produced they are accelerated and eventually escape the coma. We describe and interpret the dynamics of the cometary ion flow, of an intermediate active comet, very close to the nucleus and in the terminator plane.

Methods. We analysed in situ ion and magnetic field measurements, and characterise the velocity distribution functions (mostly using plasma moments). We propose a statistical approach over a period of one month.

Results. On average, two populations were observed, separated in phase space. The motion of the first is governed by its interaction with the solar wind farther upstream, while the second one is accelerated in the inner coma and displays characteristics compatible with an ambipolar electric field. Both populations display a consistent anti-sunward velocity component.

Conclusions. Cometary ions born in different regions of the coma are seen close to the nucleus of comet 67P/ChuryumovGerasimenko with distinct motions governed in one case by the solar wind electric field and in the other case by the position relative to the nucleus. A consistent anti-sunward component is observed for all cometary ions. An asymmetry is found in the average cometary ion density in a solar wind electric field reference frame, with higher density in the negative (south) electric field hemisphere. There is no corresponding signature in the average magnetic field strength.
\end{abstract}

Key words. comets: general - comets: individual: 67P/Churyumov-Gerasimenko - plasmas - methods: observational space vehicles: instruments

\section{Introduction}

Comets are, thanks to their enormous tails, the smallest bodies in the solar system observable from Earth with the naked eye (Yeomans et al. 1986). To be able to leave such a strong trail, comets constantly lose surface matter during their active periods. At some heliocentric distance the sublimated cometary gas together with dust starts to form a thin and gravitationally unbound atmosphere, which then gets ionised mostly by photoionisation, charge exchange, and electron impact ionisation. In contrast with most bodies in the solar system, this partially ionised atmosphere is continuously escaping. The dust and ions form two distinct tails behind the comet. The dust tail generally points away from the Sun, but is slightly curved following the direction of the cometary orbit, while the ion tail is formed closer to the anti-sunward direction through a complex and highly variable interaction with the solar wind (Alfvén 1957).

The swiftly variable cometary environment depends strongly on the distance to the Sun. The Rosetta mission (Glassmeier et al. 2007a) has given us a unique opportunity to observe the intricate changes of comet 67P/Churyumov-Gerasimenko (67P) and its environment from its arrival to the comet in August 2014 until the end of the mission in September 2016. Rosetta had the great advantage of orbiting the comet for more than $2 \mathrm{yr}$, as opposed to the short flybys of all previous cometary exploration missions. All mission overviews of the comet environment have been given by Nilsson et al. (2017) and Goldstein et al. (2017) for the ion environment and by Goetz et al. (2017) for the magnetic field environment. It is useful to differentiate between three regimes of cometary activity. At low activity the cometary ions act almost like test particles, with only a modest influence on the solar wind (Nilsson et al. 2015a; Behar et al. 2016a; Goldstein et al. 2015). During the intermediate activity period the influence on the solar wind is much more pronounced (Behar et al. 2016a; Broiles et al. 2015), but the solar wind still permeates all of the coma. At high activity the solar wind-comet atmosphere interaction becomes less linear and a solar wind ion cavity forms (Behar et al. 2017; Nilsson et al. 2017).

The newly born ions in the thin atmosphere of the comet at low and intermediate activity interact with the solar wind through the mass-loading mechanism (Szegö et al. 2000; Behar et al. 2016a, 2017). The fast solar wind with the frozen-in magnetic field accelerates the slow cometary ions in the direction determined by the solar wind flow and the surrounding magnetic field. Energy and momentum for this acceleration is taken from the solar wind flow, which is correspondingly slowed down and 
deflected. Cometary ions accelerated by the solar wind electric field, are termed pick-up ions, as they are picked up by the solar wind flow.

Pick-up cometary protons were observed at a distance of $\sim 7.8 \times 10^{6} \mathrm{~km}$ from the comet $1 \mathrm{P} /$ Halley by plasma instruments on the Giotto spacecraft. The first heavy cometary ions (e.g. water group ions) were found at a distance of $\sim 10^{6} \mathrm{~km}$ from the comet with energies of about $30 \mathrm{keV}$. Thomsen et al. (1987) report and discuss two different peaks separated in energy in the heavy cometary ion distribution function. The peak at higher energy is interpreted as particles originating upstream of the bow shock, whereas particles of the second lower energy peak are shown to originate downstream of the shock. Both populations gain their energy through their interaction with the solar wind. However, within the boundary referred to as the contact surface located $\sim 4600 \mathrm{~km}$ from the nucleus, the heavy cometary ions were observed to move radially away from the comet with a bulk velocity in the range of $1 \mathrm{~km} \mathrm{~s}^{-1}$ (Balsiger et al. 1986; Schwenn et al. 1987), showing no sign of solar wind interaction. Mass loading has been continuously observed at the comet 67P (Nilsson et al. 2017). Specifically, the case of light mass loading at comet $67 \mathrm{P}$ is described and modelled in Behar et al. (2016b).

Close to the nucleus, where the ion density is high enough, the highly mobile electrons escape from the comet much faster than heavier ions, which results in charge separation between electrons and ions. This departure from quasi-neutrality results in a radial electric field, referred to as ambipolar, slowing down the electrons and accelerating cometary ions radially outward as modelled by Vigren \& Eriksson (2017). Close to the nucleus we expect this ambipolar electric field to dominate over the solar wind electric field. Nilsson et al. (2015b) and Behar et al. (2016a) both hypothesised the existence of another polarisation electric field building up along the comet-Sun line to explain the observation of a strong anti-sunward velocity component of the cometary ions.

As the coma evolves, more processes can contribute to the plasma dynamics and Mandt et al. (2016) show that at small distances from the Sun and close to comet $67 \mathrm{P}$, collisions between neutrals and ions start to become important. The variable signatures of the cometary ions during the high activity period presented by Stenberg-Wieser et al. (2017) show the existence of short timescale dynamics.

Nilsson et al. (2015b) present observations of two cometary ion populations at the comet $67 \mathrm{P}$, separated by their energy range and flow direction: ions with an energy below about $50 \mathrm{eV}$ and coming from between the Sun and the comet direction, and a more energetic population with angle of arrival centred on the Sun direction. We now investigate the dynamics of these two populations during the comet's intermediate activity, a time period before the solar wind cavity is formed. Our goal is to understand how these two populations are formed. In particular we aim to see if one population is governed by the solar wind electric field, while the other lower energy population is governed by the ambipolar electric field of the comet ionosphere.

\section{Instrument description}

The Ion Composition Analyzer (ICA) is an imaging spectrometer, part of the Rosetta Plasma Consortium (RPC; Carr et al. 2007), designed to characterise positive ion distribution functions in the proximity of comet 67P (Nilsson et al. 2007).
The instrument provides the flow direction and energy-percharge of the ions, and is able to separate them by mass.

The field of view (FOV) has an angular coverage of $360^{\circ} \times 90^{\circ}$, corresponding to azimuth and elevation angle in the cylindrical geometry of the instrument, respectively. The azimuth angle of the incoming particles is measured by 16 azimuth anodes, covering a full circle of $360^{\circ}$ (each sector is $22.5^{\circ}$ wide). The elevation angle information is obtained by an electrostatic acceptance angle filter, consisting of two conductive plates positioned at the entrance of the instrument. By setting different voltages on the two plates, the instrument spans $\pm 45^{\circ}$ in elevation angle in 16 steps, corresponding to an angular resolution of $5.6^{\circ}$. The energy of incoming particles is determined by a toroidal electrostatic analyser (ESA), sweeping through 96 exponentially spaced steps spanning from a few eV to $40 \mathrm{keV}$. Following the ESA is a cylindrical assembly of permanent magnets creating a magnetic field that bends the trajectories of positive ions according to their momentum. The degree of deflection is identified by 32 concentric anode rings positioned at the end of the ion optics, referred to as mass anodes.

All azimuthal directions and all mass-per-charge ratios are measured simultaneously, whereas the elevation direction and energy are obtained in sweeps. The sweep over the full energy range lasts for $12 \mathrm{~s}$, which multiplied by 16 elevation steps gives the time of a full scan, $192 \mathrm{~s}$.

Through most of the mission, cometary ions are observed with energies between $20 \mathrm{eV}$ and $1 \mathrm{keV}$ (Nilsson et al. 2017); therefore, we have to consider instrumental constraints at low energies. At the energy range of tens of $\mathrm{eV}$, spacecraft charging has a great influence on the measurements, most of the time accelerating ions into the instrument due to a negative spacecraft potential. Over the duration of the Rosetta mission, the spacecraft potential varies from $-25 \mathrm{~V}$ to $+5 \mathrm{~V}$ (Odelstad et al. 2017). Additionally, the RPC-ICA has an uncertainty in the knowledge of the energy level currently believed to be about $3 \mathrm{eV}$, as well as a temperature drift of the energy scale that affects certain time periods (see Nilsson et al. 2017).

Another technical limitation arises with the elevation determination for low energy ions. Below $100 \mathrm{eV}$ the elevation resolution starts to decrease, ending at around $15 \mathrm{eV}$ with only two elevation bins. These effects are all carefully considered as explained in the following section. In addition we use the magnetic field data provided by the RPC-MAG, the magnetometer in the RPC instrument package (Glassmeier et al. 2007b).

\section{Method}

We study the dynamics of the ionised coma at low and intermediate activity. In agreement with the neutral production rates, we use the term low activity for the observational period from the arrival to the comet in August 2014 until the middle of December 2014. A period between mid-December 2014 and April 2015 is marked as the intermediate activity period (Goetz et al. 2017). After that the comet begins its high activity state, during which the mature dense coma provides a shield against the solar wind and the spacecraft is within the solar wind cavity until December 2015 (Behar et al. 2017). During the mission's outbound leg the intermediate and low activity period are observed again.

The data are inherently five-dimensional, giving the number of counts within a time period, for each energy step, mass anode, azimuth anode, and elevation step. In many cases we study only 


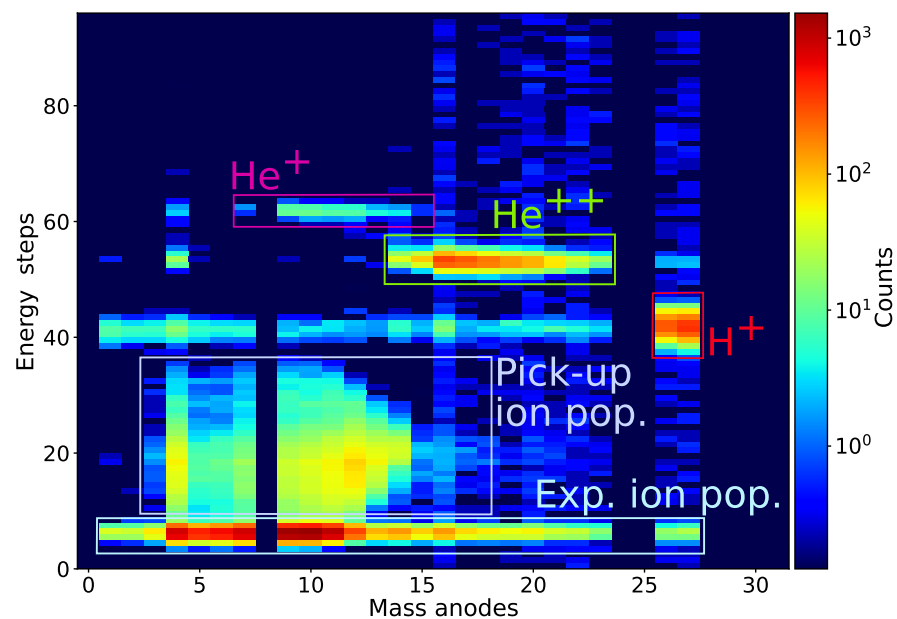

Fig. 1. Daily mass energy matrix showing an example of visual species identification for 13 January 2015. The number of counts in each pixel is colour-coded: red indicates the most counts, and blue the least or 0 . Mass anodes 0, 24, 25, 28, 29, 30, and 31 are non-operational, showing 0 counts in the plot.

two dimensions at a time, summing over all the others. For example, in the energy mass channel distribution in Fig. 1, referred to as an energy-mass matrix, each pixel is the sum of counts over one day of measurements in all viewing directions (azimuth and elevation angle) for a specified energy bin and mass anode. The signal of a single ion species appears wide and ends up on many mass anodes due to the limited resolution of the ion optics.

The positive ion species were visually identified and manually selected from the daily mass-energy matrices. An example of a mass-energy matrix from 13 January 2015 is presented in Fig. 1, where the coloured rectangles indicate the selections for each species constraining them to a number of energy steps and mass anodes. Only the full angular resolution operation modes $\left(22.5^{\circ} \times 5^{\circ}\right)$ were considered. Cometary ion data were divided into two populations, termed the expanding and the pick-up ions for the lower and higher energy ranges, respectively. The names of the two populations are explained in Sect. 5.1.

The selections were then used for the plasma moment calculations. By integrating the distribution function obtained for each scan (192s) we obtained the ion number densities and the bulk velocities for the different positive ion populations. The mean speed, directly corresponding to the observed mean energy, was also computed and used to scale the velocity vectors presented in Sect. 4.2.

The bulk velocities of the separate ion species were calculated in the instrument frame and transformed into a cometcentred solar equatorial (CSEq) frame (Behar et al. 2016a). We then performed a rotation around the comet-Sun line in order to express the ion velocities in the comet Sun electric field (CSE) frame, in which the upstream solar wind electric field is aligned with the $z$-axis. This direction is estimated from the bulk velocity of solar wind protons and alpha particles, which are deflected away from the comet-Sun line in the direction opposite to the upstream electric field. Therefore, the projection of the solar wind velocity vector in the terminator plane points towards the negative $z$-axis in the CSE frame.

We tried to estimate the direction of the upstream solar wind electric field using the magnetic field measured by the RPC-MAG. The projection of the magnetic field vector in the terminator plane is perpendicular to the direction of the upstream electric field. However, this method gives larger scatter for most purposes.

Moment calculations and frame rotations were performed for each scan during the low and intermediate comet activity, and a period of just under a month (from 26 December 2014 to 23 January 2015) is considered for the statistical analysis. The data set selection is described in Sect. 4.2.

From all scans taken during the considered period, the ones where the solar wind protons or alpha particles density is higher than the arbitrary value of $4 \times 10^{-3} \mathrm{~cm}^{-3}$ were selected because the direction of the solar wind deflection is needed to estimate the direction of the solar wind electric field. Further on, we filter the scans with the same arbitrary density limit for the signal of each cometary ion population. To avoid the inclusion of the ions affected by spacecraft potential, the maximum angular width of the expanding population peak is limited to six azimuth sectors. This is based on the assumption that the spacecraft potential attracts ions from roughly all directions, thus causing a signal seen over a wide angular space. The condition is fulfilled in $58 \%$ of the considered scans. Finally, 2606 scans were used for the statistical analysis of the expanding population, and 2672 for the pick-up population.

The data from this period is divided into 113 bins according to the measurement position in the CSE frame. The number density, the bulk velocity, and the mean speed are averaged within each bin. The calculated error values account for the statistical variations resulting from the binning process, but do not include measurement uncertainties.

\section{Observations}

\subsection{Example}

The evolution of detected positive ions was examined using daily time energy matrices illustrated by an example day, 13 January 2015 (see Fig. 2a). From the spectrogram we can use energy to differentiate the three solar wind populations (protons, alpha particles, and singly charged helium, i.e. a product of solar wind interaction with the neutral comet atmosphere; Simon Wedlund et al. 2016) and the two cometary ion populations, as marked in Fig. 2a. This species separation is confirmed by inspection of mass-matrices as discussed in the Sect. 3. The two cometary ion populations are referred to as the expanding and the pick-up populations after their measured and inferred characteristics, explained in Sect. 5.1. The expanding population appears denser with an almost constant energy span in comparison to the thinner pick-up population with an energy span that can vary as much as one order of magnitude on a daily basis.

An example of the angular distribution of the different ion species in a FOV representation of a scan taken on 13 January 2015 at 9:48:56 is plotted in Fig. 2b. The azimuth angle is shown as longitude and elevation angle as latitude.

A strong solar wind deflection can be observed: protons (red) are deflected $50^{\circ}$ away from the Sun direction (yellow), and heavier alpha particles (green) and singly charged helium (purple) are deflected by $18^{\circ}$. The pick-up ions (dark blue) are accelerated mostly anti-sunward with their bulk velocity vector pointing $21^{\circ}$ away from the comet-Sun line in the direction opposite to the solar wind deflection. These four species therefore flow in the same plane also including the comet-Sun line. The same geometry between the proton and the pick-up ion flow has been observed and explained by the mass-loading mechanism in Behar et al. (2016a). 


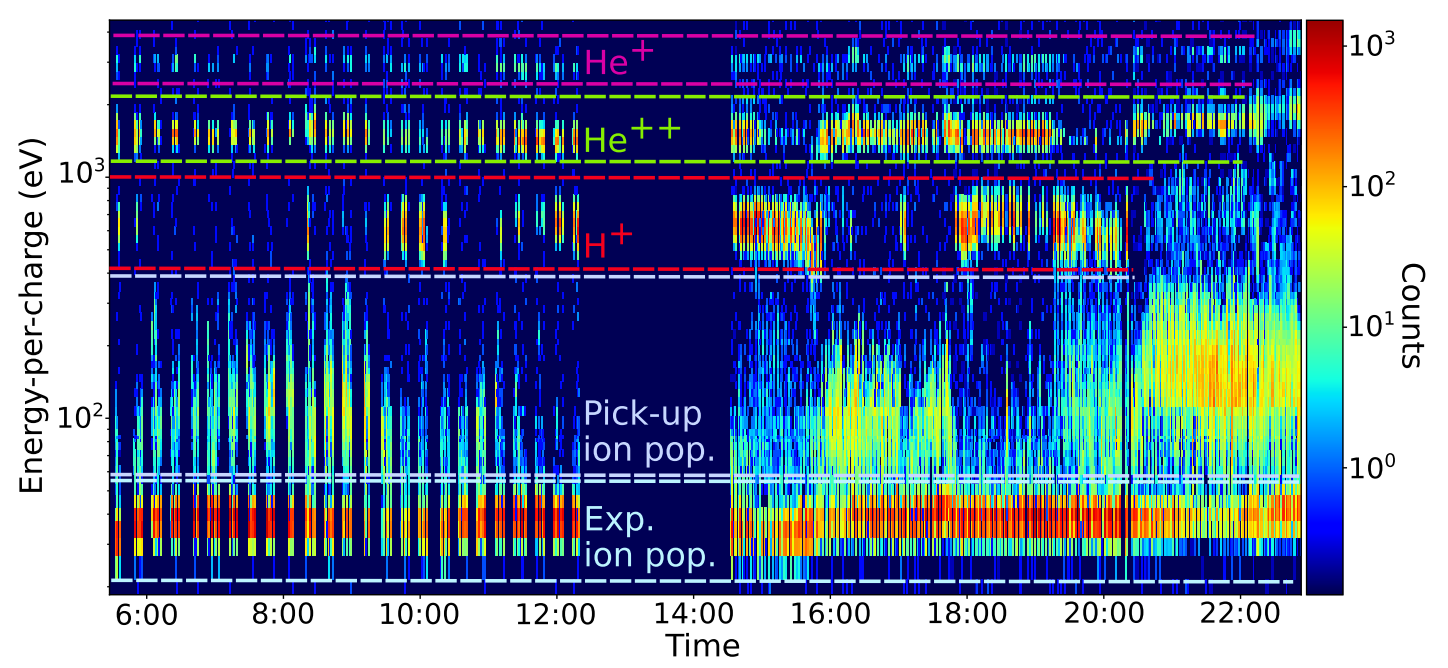

(a)

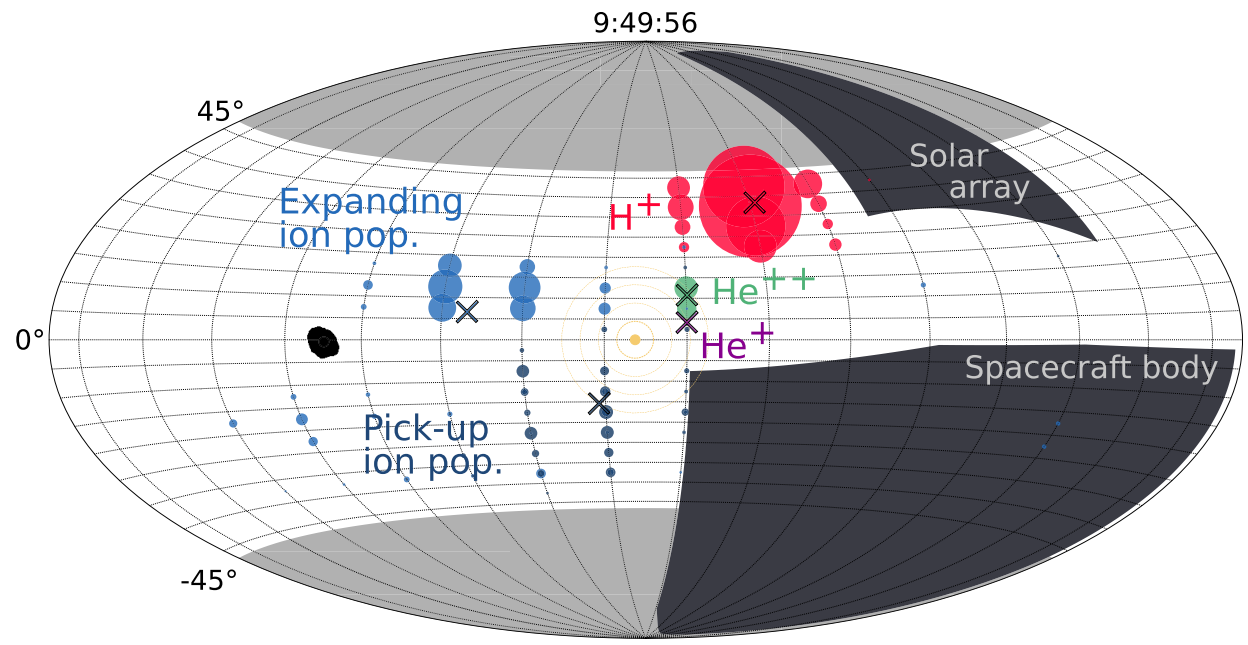

(b)

Fig. 2. Panel a: Time energy matrix (spectrogram) produced from observations made on 13 January 2015 . The marked signals at high energies are of solar origin, while at low energies we can distinguish the two ion populations: expanding and pick-up. Panel b: Scan from 13 January 2015 in a FOV representation showing arrival directions of different species. The dot sizes reflect the number of counts in each FOV unit $\left(22.5^{\circ} \times 5^{\circ}\right)$. The position of the Sun in the instrument frame is marked in yellow, and the grey areas show how the FOV is limited by the maximum elevation angles $\left(-45^{\circ}\right.$ to $\left.45^{\circ}\right)$, and obstructed by the spacecraft. In black we show the projection of the comet in the RPC-ICA field of view (ESA NavCam shape model).

Most of the expanding population ions (light blue) appear between the Sun and the comet, and some are seen at lower elevation angles and/or with the same direction as the pick-up population. The reason why we observe signal at elevations far away from the bulk of the population is most likely instrumental. For low energies, as mentioned in the instrument description, the elevation angle resolution is decreased and the ion trajectories might have been changed by the spacecraft potential. The signal appearing in the same position as the pick-up population might actually be a part of it because energy distributions of both populations sometimes overlap. However, these uncertain signals are small enough not to have a substantial impact on the calculated plasma moments (the bulk directions of each species is indicated by crosses in Fig. 2b).

\subsection{Statistical observations}

The ion environment sampled by the RPC-ICA is strongly dependent on two parameters plotted versus time in Fig. 3: the heliocentric and the cometocentric distance. Firstly, the distance from the Sun strongly affects cometary activity, and secondly the observed ion dynamics depends on the ion density, which decreases with the cometocentric distance by $1 / r_{\text {comet }}$ (Edberg et al. 2015). Thus, the time intervals with similar parameters appropriate for statistical studies are not very common. One of these few periods when the two cometary ion populations are constantly observed and clearly separated is an interval between 26 December 2014 and 23 January 2015, which was chosen for statistical analysis (see considered period marked in Fig. 3). During this time the spacecraft had a stable orbit in the comet's terminator plane with the distance between $27.5 \mathrm{~km}$ and $28.5 \mathrm{~km}$, and the comet approached the Sun from the distance of $2.7 \mathrm{AU}$ to $2.5 \mathrm{AU}$.

The average cometary ion flow is shown in Fig. 4. Dark blue and light blue coloured arrows represent the velocity vectors of respectively the pick-up and the expanding population. The pickup population flow direction in the terminator plane (left panel in Fig. 4) aligns quite well with the $z$-axis, while the expanding 


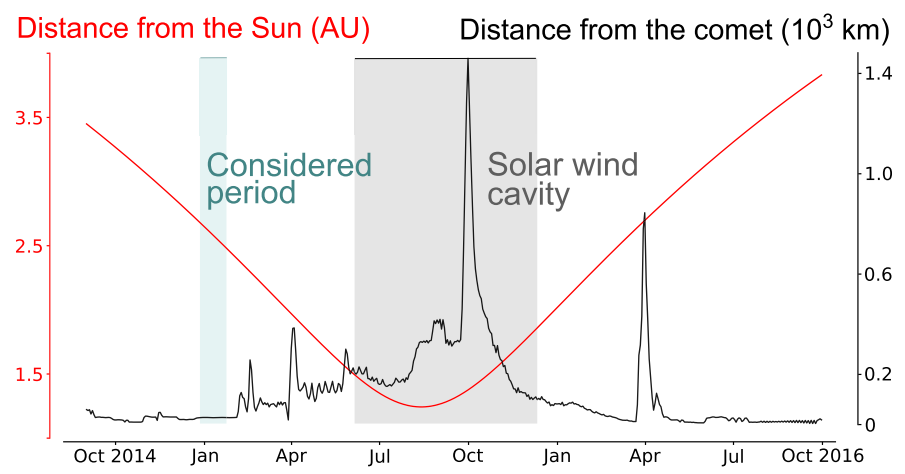

Fig. 3. Overview of the Rosetta mission period, showing the evolution of distance from the Sun and from the comet with time. Two time intervals are marked: the interval during which the spacecraft is within the solar wind cavity and the interval that was considered for the statistical analysis.

population in this plane appears to be moving radially away from the comet. In the right panel we show the cometary ion behaviour in the $x z$-plane, where both cometary ion populations exhibit a strong anti-sunward velocity component, as already noticed by Nilsson et al. (2015b), Behar et al. (2016a) and Nilsson et al. (2017).

The calculated mean speed of the expanding population during this period is $14 \mathrm{~km} \mathrm{~s}^{-1}$. Taking into consideration that these ions have been accelerated by a negative spacecraft potential of $\sim 15 \mathrm{~V}$, we estimate their velocity to $6 \mathrm{~km} \mathrm{~s}^{-1}$. The limiting values of spacecraft potential for the considered period evaluated by Odelstad et al. (2017) and Odelstad et al. (2015) are $-5 \mathrm{~V}$ and $-20 \mathrm{~V}$, therefore limiting the calculated mean speed to the interval from 0 to $12 \mathrm{~km} \mathrm{~s}^{-1}$. Similarly, the mean speed of cometary pick-up ions is observed to be $34 \mathrm{~km} \mathrm{~s}^{-1}$, estimated to be $31 \mathrm{~km} \mathrm{~s}^{-1}$ with the low and high limit at 30 and $33 \mathrm{~km} \mathrm{~s}^{-1}$, respectively.

The densities of the two cometary ion populations shown in Fig. 5a and $\mathrm{b}$ as functions of the observation location in the terminator plane, reveal an asymmetry in the cometary plasma environment. The densities of the two populations appear to be higher on the south (negative E) side of the CSE frame. The mean number density in the southern hemisphere is $16.3 \mathrm{~cm}^{-3}$ for the expanding population and $0.4 \mathrm{~cm}^{-3}$ for the pick-up population, while in the northern hemisphere the corresponding values are $9.4 \mathrm{~cm}^{-3}$ and $0.1 \mathrm{~cm}^{-3}$. The magnetic field amplitude measured by the RPC-MAG is binned in the same way as the density, and is given in Fig. 5c. No correlation between this strength and the position angle in CSE coordinates is found.

\section{Discussion}

\subsection{Pick-up and expanding populations}

Our observations reveal the existence of two cometary ion populations, distinguishable by their energy, flow direction, and density.

The pick-up ion population is more energetic and directionally ordered by the observed solar wind, as described in the previous section. Both the pick-up and the solar wind ions on average exhibit no $v_{y}$-component in the CSE frame, and a $v_{z}$-component of opposite sign to each other. The dynamics of this interaction can be explained by the mass-loading mechanism. Far from the comet, the solar wind is little disturbed by the tenuous coma and largely dominates the plasma environment.
This fast solar wind together with the frozen-in interplanetary magnetic field (IMF) exert an electric field on the slow new born cometary ions.

The acceleration of each ion is ruled by the Lorentz force

$\boldsymbol{a}_{\mathrm{i}}=\frac{q}{m}\left(\boldsymbol{E}+\boldsymbol{v}_{\mathrm{i}} \times \boldsymbol{B}\right)$,

where $\boldsymbol{B}$ is the IMF, $\boldsymbol{v}_{\mathrm{i}}$ is the ion velocity, and $\boldsymbol{E}$ is the total electric field. Neglecting pressure gradients, collisions, currents, and resistivity, this total electric field is reduced to the solar wind electric field, given by the cross product between the bulk velocity of all ions $(\boldsymbol{u})$ and magnetic field: $\boldsymbol{E}_{S W}=-\boldsymbol{u} \times \boldsymbol{B}$. We can rewrite the equation of motion as

$\boldsymbol{a}_{\mathrm{i}}=\frac{q}{m}\left(\boldsymbol{v}_{\mathrm{i}}-\boldsymbol{u}\right) \times \boldsymbol{B}$.

The total ion bulk velocity of a plasma consisting of equally charged solar wind and cometary ions is

$\boldsymbol{u}=\frac{n_{\mathrm{sw}} \boldsymbol{v}_{\mathrm{sw}}+n_{\mathrm{com}} \boldsymbol{v}_{\mathrm{com}}}{n_{\mathrm{sw}}+n_{\mathrm{com}}}$,

and thus strongly depends on the number density $(n)$ of each population.

We first consider an extreme condition. Far away from the comet on the day-side, upstream of the measurement point where the coma is extremely thin, we can assume that the number density ratio $n_{\mathrm{com}} / n_{\mathrm{sw}}$ will tend to 0 . The total ion bulk velocity is then equal to the bulk velocity of the solar wind ions $\left(\boldsymbol{u}=\boldsymbol{v}_{\mathrm{sw}}\right)$. The newly born ions with initial velocity close to 0 get accelerated in the direction perpendicular to both $\boldsymbol{v}_{\mathrm{sw}}$ and $\boldsymbol{B}$, and the solar wind continues almost undisturbed.

Closer to the nucleus, the number density ratio increases, and the total ion bulk velocity also starts depending on the bulk velocity of the cometary ions. As soon as we consider the motion of these ions, the acceleration $\boldsymbol{a}_{\mathrm{i}}$ they experience is no longer perpendicular to the solar wind velocity. Because all cometary ions are created with no initial velocity (compared to the high speed of the solar wind), the acceleration $\boldsymbol{a}_{\mathrm{i}}$ and the total ion bulk velocity $\boldsymbol{u}$ remain in the same plane, assuming that the IMF is perpendicular to the solar wind flow. At the same time the solar wind feels an acceleration in the opposite direction and gets deflected from its flow direction. This increase in density ratio $n_{\text {com }} / n_{\text {sw }}$ also corresponds to a decrease in the total ion bulk velocity $\boldsymbol{u}$. Consequently, the frozen-in magnetic field piles up and contributes to the complexity of the ion dynamics, in the case of comparable number densities. During the considered period the average number density ratio is 0.83 . The bulk velocity of the pick-up ions shows a strong anti-sunward component, but remains in the same plane as the deflected solar wind flow: without the $v_{y}$-component and with the oppositely signed $v_{z}$-component.

The intricacy of the mass-loading mechanism in connection to the similar observations of the strong anti-sunward component in the flow direction of the cometary ions has already been addressed by Behar et al. (2016a) in a case study of a day when 67P was at low activity, and by Nicolaou et al. (2017) who give two examples from the medium and high activity period.

In order to interpret our observations we depict a region far upstream of the nucleus where the behaviour of electric and magnetic fields results only from the relative motion of the different ion populations in the absence of any currents or charge separation. Because of the ions' high energy and consistent orientation with respect to the solar wind, we conclude that the flow pattern 

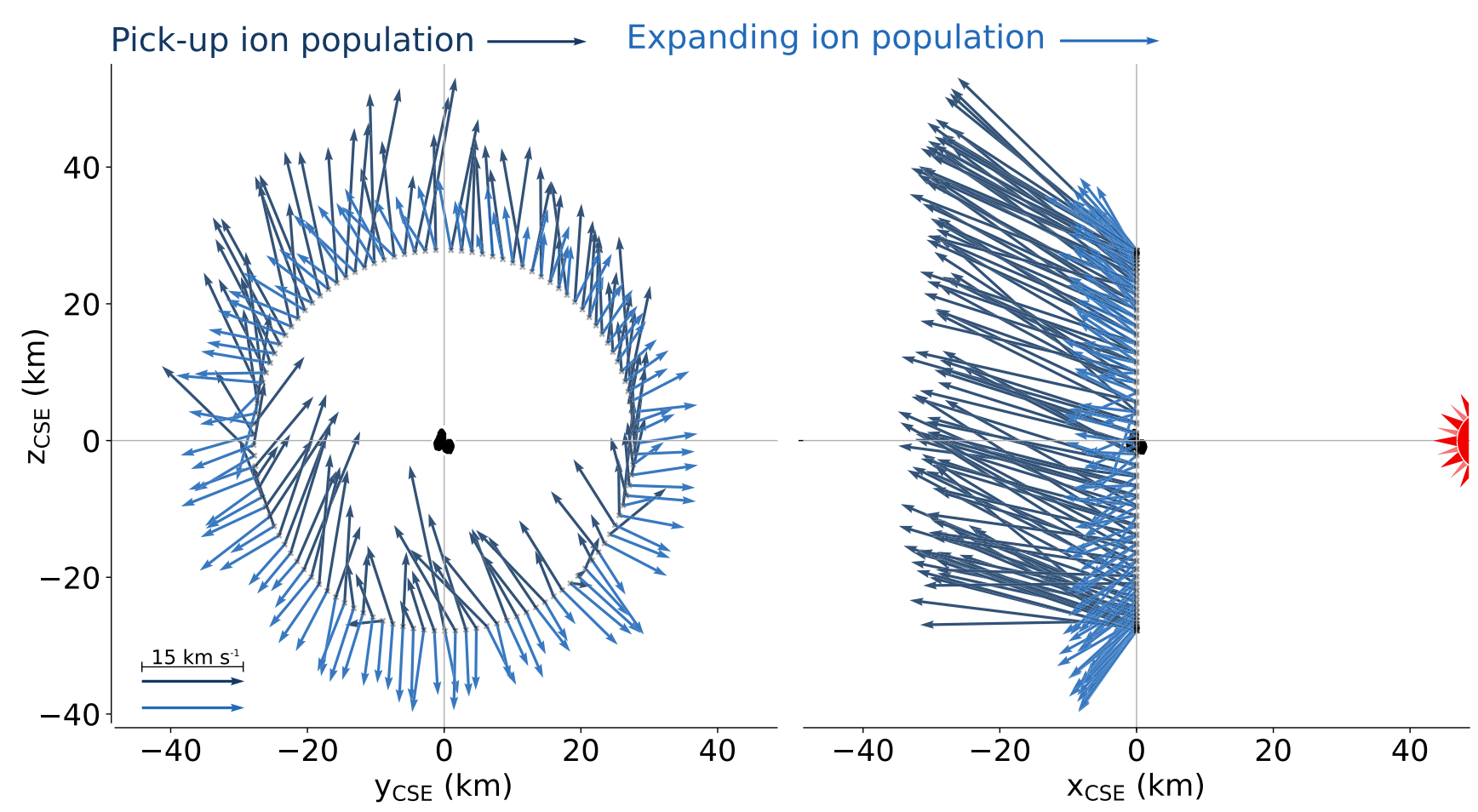

Fig. 4. Velocity vectors of the pick-up and expanding cometary ion populations, indicated in different colours. We show two planes in the CSE frame: on the left the terminator plane (yz-plane) and on the right the $x z$-plane.

of the pick-up ions is mainly a consequence of the mass-loading mechanism. Even though the anti-sunward velocity component of this population could be the result of this same mechanism, we do not exclude the possibility of another force additionally accelerating them away from the Sun.

The expanding population flows radially outward and antisunward, symmetrically around the comet-Sun line. The expanding cometary ions, in contrast to the pick-up ions, show no correlation between the direction of their flow and the solar wind. Their energies are typically $\sim 10$ times smaller than the energies of the pick-up ions and the density on the other hand is 10-100 times higher. This leads us to believe that the two populations originate from different regions within the coma. The expanding population originates from a dense region, closer to the comet and to the observation point. Ions are born from the neutral molecules expanding radially away from the comet with a velocity of not more than $1 \mathrm{~km} \mathrm{~s}^{-1}$ (Vigren \& Eriksson 2017). In the short time between their birth and point of detection, expanding population ions get accelerated on average to $6 \mathrm{~km} \mathrm{~s}^{-1}$, and gain a strong anti-sunward component.

The radial acceleration process of cometary ions was modelled in 1D by Vigren \& Eriksson (2017) assuming the presence of a constant electric field in the range of $0.1 \mathrm{mV} \mathrm{m}^{-1}$. This electric field can be explained as follows (Vigren et al. 2015): close to the nucleus, the density of cometary ions is high and decreases with $1 / \mathrm{r}_{\text {comet }}$, as described in a simple model proposed by Haser (1957). The decrease in the plasma density with distance from the comet results in a radial pressure gradient for both the ions and the electrons. The lighter electrons escape the nucleus faster then the heavier ions, which results in a charge separation. This imbalance gives rise to an electric field, referred to as an ambipolar electric field, directed radially away from the nucleus. This field accelerates the slowly expanding water group ions and decelerates the fast escaping electrons.
Assuming an isothermal coma where the electron number density linearly decreases with the cometocentric distance $r$, we obtain an expression for the radial, ambipolar electric field (Vigren et al. 2015),

$E_{\mathrm{r}}=-\frac{1}{n_{\mathrm{e}} q} \frac{d p_{\mathrm{e}}}{d r}=\frac{k_{\mathrm{B}} T_{\mathrm{e}}}{q r}$,

where $k_{\mathrm{B}}$ is the Boltzman constant, $T_{\mathrm{e}}$ electron temperature, and $q$ the ion charge. The acceleration of an ion at a distance $\mathrm{r}$ from the comet is then given by

$a_{r}(\mathrm{r})=\frac{\mathrm{qE}_{\mathrm{r}}}{\mathrm{m}_{\mathrm{com}}}=\frac{\mathrm{k}_{\mathrm{B}} \mathrm{T}_{\mathrm{e}}}{\mathrm{m}_{\mathrm{com}} r}$

with $m_{\text {com }}$ being the mass of the accelerated ions. During the considered period the typical value of electron temperature was $5 \mathrm{eV}$ [Eriksson et al, 2017], and the distance from the comet $28 \mathrm{~km}$, resulting in a radial electric field of $0.2 \mathrm{mV} \mathrm{m}^{-1}$. Accordingly, the singly charged cometary water ions undergo an acceleration of $1 \mathrm{~km} \mathrm{~s}^{-2}$.

In this model it is assumed that the heavy ions are largely dominating within the inner coma, and will therefore not be affected by the solar wind. However, as observed in Behar et al. (2016a) and Nilsson et al. (2017), and in the present work, a consistent anti-sunward direction appears in both cometary populations, including the expanding population. This is discussed by Nilsson et al. (2015b) and Behar et al. (2016a) in terms of an additional electric field that could exist on a macroscopic scale, accelerating all cometary ions in the anti-sunward direction. The basic idea is that for a comet ionosphere much smaller than the pick-up ion gyroradius, a polarisation electric field will arise due to the different motion of ions and electrons, for example as described in a barium release context by Haerendel et al. (1986) and Brenning et al. (1991). 


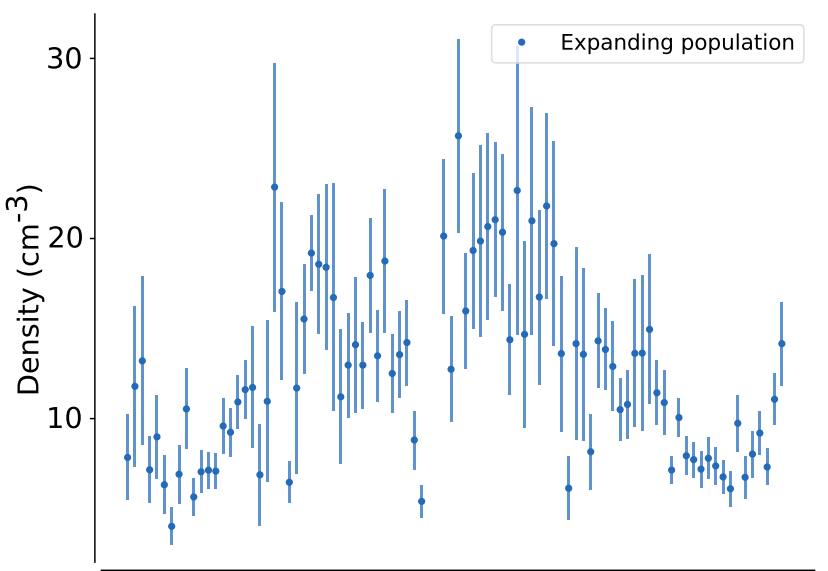

(a)

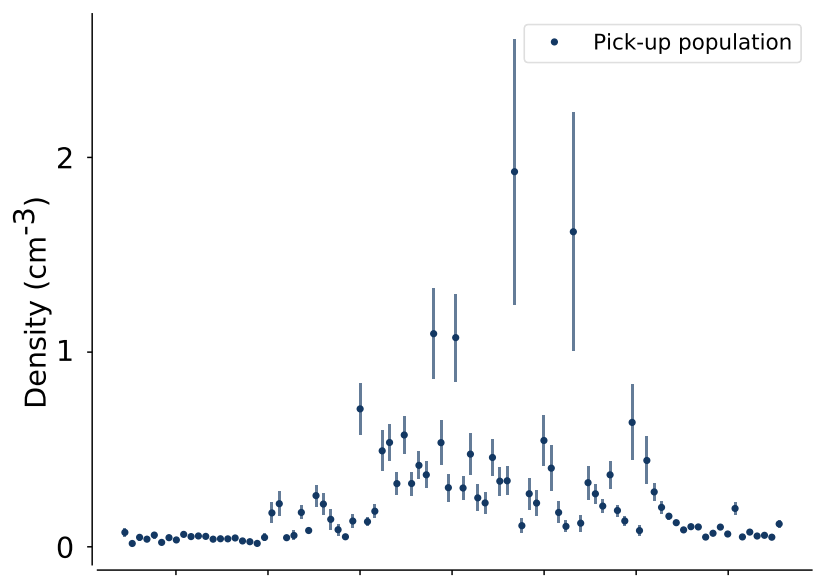

(b)

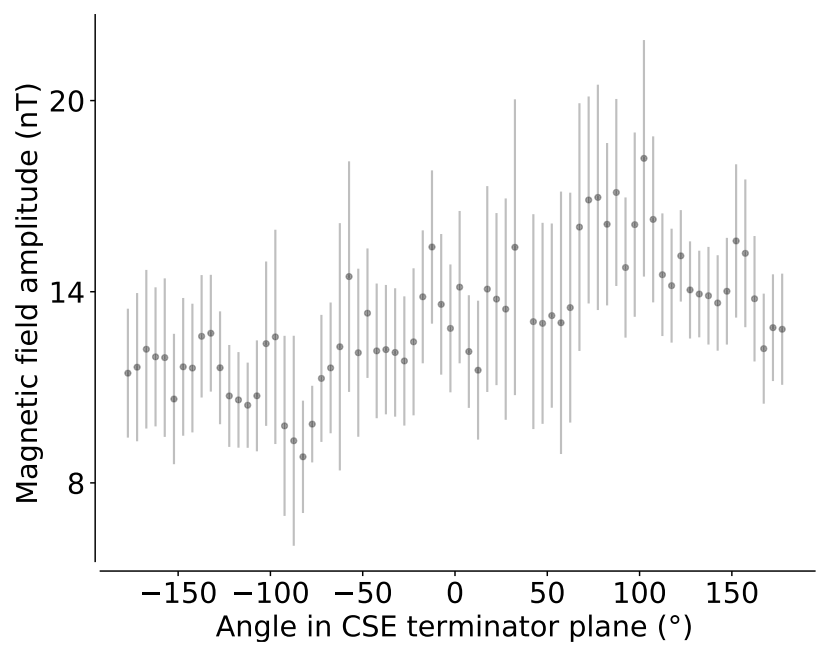

(c)

Fig. 5. Density of $(a)$ the expanding and $(b)$ the pick-up population plotted against the angular position in CSE frame. Angle $0^{\circ}$ corresponds to the south electric field pole direction (negative $z$ ) and angle $\pm 180^{\circ}$ to the north pole direction. Panel $c$ : Magnetic field amplitude measured by the RPC-MAG with relation to the angular position in CSE frame.

A summary of the inferred motion of the different ions is presented in Fig. 6. We concluded that the pick-up ions originate from a region upstream of the measurement point (dark blue region) dominated by the solar wind, and the expanding ion population originates from a region closer to the nucleus than the observation point (light blue region) and accelerated by both

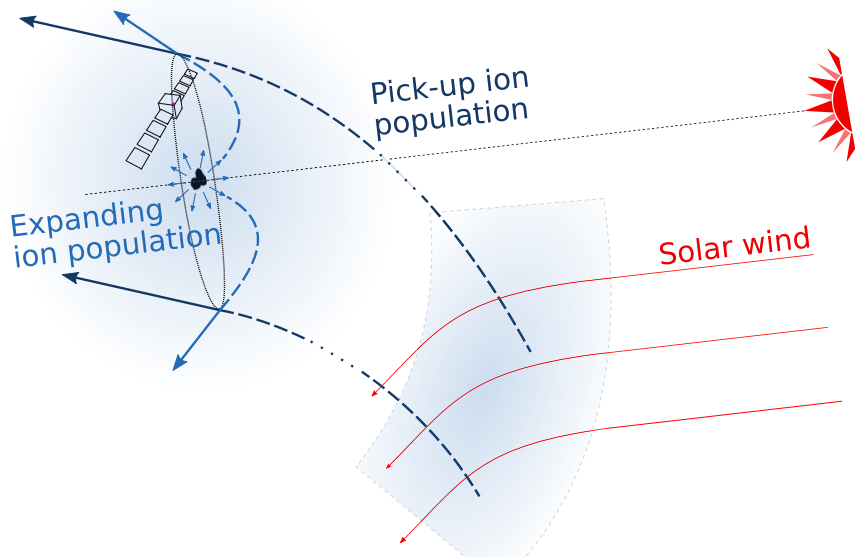

Fig. 6. Schematics showing the inferred ion trajectories for both cometary populations. Dark blue marks the area of birth of the observed pick-up ions that are accelerated through the interaction with the solar wind. The light blue area represents the region of birth of the observed expanding ion population, which is dominated by the cometary ions. Also shown is the Rosetta spacecraft in a terminator plane orbit around the comet.

an ambipolar electric field and an additional anti-sunward electric field. For the expanding population this would result in a cylindrical geometry outlining the fountain flow, as shown in the schematics. The flow obviously reminds us of the fountain model proposed by (Biermann et al. 1967; see schematics in their Fig. 1). This model is now outdated, as the solar wind interaction with comet $67 \mathrm{P}$ is believed to be uncollisional during the considered period (Mandt et al. 2016). Furthermore, no boundaries were observed at the comet and the solar wind entirely permeates the coma during the period of low and medium activity (Behar et al. 2017), thus in our case a force other than viscosity is necessary to drag the radial outflow downstream.

\subsection{Density asymmetry}

Another surprising aspect of the ion dynamics appears in the observations. The densities of both cometary ion populations are on average higher in the southern hemisphere in the CSE frame than in the northern. In many simulation works the opposite is seen. For instance Deca et al. (2017); Rubin et al. (2014) and Koenders et al. (2016) addressing heliocentric distances of respectively 3.0, 2.7, and $2.0 \mathrm{AU}$, observe that the orientation of the upstream electric field, pointing northward in the CSE frame, shifts the positive cometary ions northward as well. In these three articles, an asymmetry is seen between the northern and the southern hemispheres in the heavy ion density, but with the opposite orientation, namely higher densities are seen in the northern hemisphere. In our interpretation concerning the expanding population, no asymmetry would be expected.

The illumination of the comet's irregular shape cannot be the reason for this pattern either. The nucleus orientation is constantly and randomly changing in the CSE frame of reference, just as the upstream electric field changes its direction randomly in the CSEq frame.

Density patterns are observed and follow the orientation of the upstream electric field, without observable counterparts in the local magnetic field, measured at the spacecraft location. These electric field asymmetries are thus a result yet to be explained, with the possible need for higher resolution simulations and more detailed physics close to the nucleus. 


\subsection{Cometary ion dynamics out of the selected period}

The observed cometary ion energies and fluxes varied strongly through the mission and different dynamics regimes are expected at different distances from the comet and/or from the Sun (Nilsson et al. 2017).

The first cometary ions were observed on 8 August 2014, and their flow direction suggests that they were the pick-up ions. Beginning with 21 September 2014, both the pick-up and the expanding populations of cometary ions were frequently observed and clearly separable (Nilsson et al. 2015a). During this low activity period until 5 February 2015, the ion dynamics is fairly similar to that described above. However, the stability of the orbit parameters (see Fig. 3 for the cometocentric distance) and the good time coverage during the considered period made it a better data set for statistic studies. For safety reasons, after 5 February the spacecraft moved away from the comet to a distance of about $100 \mathrm{~km}$ because the comet's activity increased. Starting from this point and until perihelion the density of accelerated ions continues to grow (Nilsson et al. 2017). The expanding population can be recognised from the observations reported by Stenberg-Wieser et al. (2017) at greater distances from the comet $(>100 \mathrm{~km})$ during the day-side excursion. They also reveal new cometary ion features on short timescales, as the instrument started its first measurements in a new high time resolution mode in May 2015.

The comet goes through intermediate to low activity again on the mission outbound leg, but sampling parameters - the heliocentric and the cometocentric distance - are never comparable to those from the beginning of the mission. When the comet again reaches a distance of $2.6 \mathrm{AU}$ from the Sun (comparable to the considered period), the spacecraft starts an excursion towards the coma night-side, probing distances of up to $1000 \mathrm{~km}$ from the comet. This provides a great opportunity to complement the current study of the cometary ion dynamics as ions in the terminator plane eventually reach the night-side of the coma. However, the expanding population throughout most of the low and medium comet activity period exhibits constant characteristics in scope of energy and direction (Nilsson et al. 2017).

\section{Conclusion}

In this article we provide observations of the cometary ion dynamics and their interpretation for a medium activity comet (between 2.5 and 2.7 AU from the Sun) and in the terminator plane, at a distance of $\sim 28 \mathrm{~km}$ from the comet.

In this regime we identify two cometary ion populations, namely the pick-up population and the expanding population. The first has gained most of its energy and momentum through its interaction with the solar wind farther upstream of the observation point. Its direction of motion in the CSE $y z$-plane is essentially in the positive $z$ direction, i.e. along the solar wind electric field direction. The motion of this population is thus strongly governed by the solar wind electric field.

The second population gained most of its energy in the close vicinity of the nucleus where cometary ions largely dominate and where the acceleration appears to be driven by an ambipolar electric field. The ion motion in the $y z$-plane is radially away from the comet independent of the solar wind electric field direction. This population thus comes from a part of the coma where the local ambipolar electric field governs the ion motion, and the solar wind electric field has much less influence.

In agreement with Behar et al. (2016a) and Nilsson et al. (2017), the present observations point towards the existence of an additional electric field accelerating all cometary ions towards the anti-sunward direction. The data thus suggest, that there is a third electric field in the vicinity of the comet, in addition to the solar wind and ionospheric ambipolar electric fields, affecting both the cometary ion populations.

The density of the two cometary ion populations reveals an intrinsic asymmetry of the coma of 67P, with higher densities in the southern CSE hemisphere. This shows that the solar wind electric field does have some influence on the expanding population, even though not much influence is seen in the flow direction.

Acknowledgements. The work on RPC-MAG was financially supported by the German Ministerium für Wirtschaft und Energie and the Deutsches Zentrum für Luft- und Raumfahrt under contract 50QP 1401. We acknowledge the staff of CDDP and IC for the use of AMDA and the RPC Quicklook data base, which was provided by a collaboration between the Centre de Données de la Physique des Plasmas (CDPP) supported by CNRS; CNES; Observatoire de Paris and Université Paul Sabatier, Toulouse; and Imperial College London, supported by the UK Science and Technology Facilities Council. We are indebted to the whole Rosetta mission team, Science Ground Segment, and Rosetta Mission Operation Control for their hard work in making this mission possible. The spice toolkit from NASA's Navigation and Ancillary Information Facility was used in this study, and was made accessible through Python by the SpiceyPy wrapper.

\section{References}

Alfvén, H. 1957, Tellus, IX, 92

Balsiger, H., Altwegg, K., Buhler, F., et al. 1986, Nature, 321, 330

Behar, E., Nilsson, H., Wieser, G. S., et al. 2016a, Geophys. Res. Lett., 43, 1411 Behar, E., Lindkvist, J., Nilsson, H., et al. 2016b, A\&A, 596, A42

Behar, E., Nilsson, H., Alho, M., Goetz, C., \& Tsurutani, B. 2017, MNRAS, 469, S396

Biermann, L., Brosowski, B., \& Schmidt, H. U. 1967, Sol. Phys., 1, 254

Brenning, N., Kelley, M. C., Swenson, C., Providakes, J., \& Stenbaek-Nielsen, H. C. 1991, J. Geophys. Res., 96, 9735

Broiles, T. W., Burch, J. L., Clark, G., et al. 2015, A\&A, 583, A21

Carr, C., Cupido, E., Lee, C. G. Y., et al. 2007, Space Sci. Rev., 128, 629

Deca, J., Divin, A., Henri, P., et al. 2017, Phys. Rev. Lett., 118, 205101

Edberg, N. J. T., Eriksson, A. I., Odelstad, E., et al. 2015, Geophys. Res. Lett., 42,4263

Glassmeier, K.-H., Boehnhardt, H., Koschny, D., Kührt, E., \& Richter, I. 2007a, Space Sci. Rev., 128, 1

Glassmeier, K.-H., Richter, I., Diedrich, A., et al. 2007b, Space Sci. Rev., 128, 649

Goetz, C., Volwerk, M., Richter, I., \& Glassmeier, K.-H. 2017, MNRAS, 469, $\mathrm{S} 268$

Goldstein, R., Burch, J. L., Mokashi, P., et al. 2015, Geophys. Res. Lett., 42, 3093 Goldstein, R., Burch, J. L., Mokashi, P., et al. 2017, MNRAS, 469, S262

Haerendel, G., Paschmann, G., Baumjohann, W., \& Carlson, C. W. 1986, Nature, 320,720

Haser, L. 1957, Bull. Cl. Sci. Acad. Roy. Belg., 43, 740

Koenders, C., Goetz, C., Richter, I., Motschmann, U., \& Glassmeier, K.-H. 2016, MNRAS, 462, S235

Mandt, K. E., Eriksson, A., Edberg, N. J. T., et al. 2016, MNRAS, 462, S9

Nicolaou, G., Behar, E., Nilsson, H., et al. 2017, MNRAS, 469, S339

Nilsson, H., Lundin, R., Lundin, K., et al. 2007, Space Sci. Rev., 128, 671

Nilsson, H., Stenberg Wieser, G., Behar, E., et al. 2015a, Science, 347, 0571

Nilsson, H., Stenberg Wieser, G., Behar, E., et al. 2015b, A\&A, 583, A20

Nilsson, H., Wieser, G. S., Behar, E., et al. 2017, MNRAS, 469, S252

Odelstad, E., Eriksson, A. I., Edberg, N. J. T., et al. 2015, Geophys. Res. Lett.,

$$
42,126
$$

Odelstad, E., Stenberg-Wieser, G., Wieser, M., et al. 2017, MNRAS, 469, S568

Rubin, M., Koenders, C., Altwegg, K., et al. 2014, Icarus, 242, 38

Schwenn, R., Ip, W. H., Rosenbauer, H., et al. 1987, A\&A, 187, 160

Simon Wedlund, C., Kallio, E., Alho, M., et al. 2016, A\&A, 587, A154

Stenberg-Wieser, G., Odelstad, E., Wieser, M., et al. 2017, MNRAS, 469, S522

Szegö, K., Glassmeier, K.-H., Bingham, R., et al. 2000, Space Sci. Rev., 94, 429

Thomsen, M. F., Feldman, W. C., Wilken, B., Jockers, K., \& Stuedemann, W. 1987, A\&A, 187, 141

Vigren, E., \& Eriksson, A. I. 2017, ApJ, 153, 150

Vigren, E., Galand, M., Eriksson, A. I., et al. 2015, ApJ, 812, 54

Yeomans, D. K., Rahe, J., \& Freitag, R. S. 1986, JRASC, 80, 62 